\title{
IS RETROGRADE INTRARENAL SURGERY PREFEREABLE OVER MINI PERCUTANEOUS NEPHROLITHOTOMY IN PATIENTS WITH RENAL CALCULUS OF LESS THAN OR EQUAL TO 2 CM SIZE?
}

\section{Girish H M}

\section{Sanman K N* \\ G G Laxman \\ Prabhu}

\section{Ranjit Shetty}

DNB Registrar -Department of Urology, Kasturba Medical College ( A unit of Manipal Academy of Higher Education), Mangaluru.

DNB (Genito-Urinary surgery/Urology) -Assistant Professor, Department of Urology, Kasturba Medical College (A Unit of Manipal Academy of Higher Education),Mangaluru. *Corresponding author

Mch (Urology) - Professor, Department of Urology, Kasturba Medical College (A Unit of Manipal Academy of Higher Education), Mangaluru.

Mch (Urology) - Associate Professor, Department of Urology, Kasturba Medical College (A Unit of Manipal Academy of Higher Education), Mangaluru.

ABSTRACT OBJECTIVE: To compare intraoperative and post-operative outcomes between mini-percutaneous Nephrolithotomy (miniPCNL) and Retrograde intrarenoscopy (RIRS) groups.

MATERIALS AND METHODS: A total of 66 patients were alternatively allocated to undergo miniPCNL (33patients, group A) and RIRS (33patients, group B) groups. All patients were evaluated by history, laboratory investigations, X-ray Kidney ureter bladder (KUB), Ultrasonography and computerized tomography (CT) scan to determine stone number, location and size. Operative time, stone free rate, duration of hospital stay and complications were compared between both groups.

RESULTS: Demographic parameters were comparable between two groups. Stone free rate was similar in both groups. Mean duration of procedure was less for miniPCNL when compared to RIRS with $\mathrm{P}<0.0001$. Duration of post-operative stay for miniPCNL was longer when compared to RIRS with $\mathrm{P}<0.0001$. Complications like intra operative bleeding, post-operative fever and blood transfusion were similar in both groups. The RIRS group patients experienced reasonably less pain postoperatively as per Visual analogue scale (VAS) score when compared to miniPCNL group with $\mathrm{P}<0.0001$.

CONCLUSION: Though RIRS and miniPCNL have good and comparable stone free rate with low complication rates, favourable pain score and less hospital stay makes RIRS a preferred option over miniPCNL.

KEYWORDS :Retrograde Intrarenal Surgery, VAS Score, Mini-percutaneous Nephrolithotomy, Stone-free Rate.

\section{INTRODUCTION}

Incidence of urolithiasis is increasing globally as a common disease ailment, which has considerable social and economic implications in the society. ${ }^{[1,2]}$ Last four decades has witnessed considerable evolution in management of renal calculi. ${ }^{[1]}$ The treatment modalities have been and being modified continuously to improvise the efficacy along with minimization of complications. Percutaneous nephrolithotomy (PCNL), retrograde intrarenal surgery (RIRS) and shockwave lithotripsy (SWL) are the current management options for renal calculi. ${ }^{[3]}$

Fernstrom and Johansson described PCNL for the first time in 1976, which became procedure of choice for large and small renal calculi. ${ }^{[3,4]}$ Though stone clearance is good in PCNL, there are significant complications associated with the procedure. ${ }^{[5]}$ Most of the complications such as bleeding, tear of infundibulum or calyx, urine leak and nephrons loss are mainly attributable to tract size in PCNL. ${ }^{[6,7]}$ Bleeding still remains a significant morbidity with $\mathrm{PCNL} .{ }^{[5,6]}$ While most bleeding associated with PCNL can be managed conservatively, approximately $0.6-1.4 \%$ of patients requires angioembolization to control intractable bleeding. ${ }^{[8]}$ Traditionally, PCNL required a 24-30 Fr nephrostomy sheath for renal access. With the development of smaller sheaths, it was found that mini-PCNL (12-20F sheath) could be performed with minimal damage to renal parenchyma, thereby reducing the procedure related morbidity without diminishing its therapeutic efficacy. ${ }^{[7,9]}$

RIRS is a minimally invasive treatment modality used to treat intrarenal calculi with the potential to decrease morbidity, while maintaining a high level of efficacy. Advancement in ureteroscopy has now enabled unrestricted access to calculi at virtually all locations in the urinary tract. ${ }^{[10]}$
Currently, there are only a few prospective studies comparing safety and efficacy of RIRS and miniPCNL for renal calculi less than $2 \mathrm{~cm}$ in size. This study is aimed a multi parametric comparison of miniPCNL and RIRS, in patients with renal calculus of less than or equal to $2 \mathrm{~cm}$ in size.

\section{MATERIALS AND METHODS}

This is a prospective observational study which was conducted from January 2018 to April 2019, after obtaining study protocol approval from institutional ethical committee on human research.(IEC number: KMC MLR 12-17/255). Written Informed consent was taken from all subjects before starting the study. Total 66 patients were included in the study were alternatively allocated to undergo miniPCNL (group A) and RIRS (group B). 33 patients underwent miniPCNL (group $A$ ) and 33 patients underwent RIRS (group B). Patients with age $<18$ years, multiple stones, stone size more than $2 \mathrm{~cm}$, patients with anomalous kidney, uncorrected coagulopathies and pregnant women were excluded from the study.

After obtaining detailed history, standard physical examinations were performed before surgery. Apart from routine hematological investigations, all patients were evaluated with X-ray kidney ureter bladder (KUB), Ultrasonography (USG) and computed tomography (CT) to determine the exact size, number and location of the renal stones.

The following parameters were compared between the two groups, namely size and location of the stones as well as age, gender, need for blood transfusion, complication rates, duration of procedure, post-operative pain, postoperative hospital stay, stone clearance and need for auxiliary procedures. 
Stone free rate (SFR): Defined as no stone visible on $\mathrm{X}$ ray KUB and ultrasonography after the procedure. Post operatively at 1 month, ultrasound scan showing residual fragments smaller than $4 \mathrm{~mm}$ was designated as clinically insignificant residual fragment.

Operative time in minutes and duration of hospital stay in days were compared in both groups. Both the procedures were performed under general anesthesia.

Visual analog scale score was used to objectively grade postoperative pain.

mini-PCNL: Cystoscopy was done in lithotomy position to place a $5 \mathrm{Fr}$ ureteric catheter in the affected side ureter and patient was catheterized. MiniPCNL was performed by in prone position with fluoroscopic imaging guidance and single step dilatation done to place a 16.5Fr sheath. A $12 \mathrm{Fr}$ Nephroscope (Karl Storz, Germany) with 12 degree angle was used to visualize the stones, which were dusted using Holmium laser system ( $365 \mu \mathrm{m}$ fibre, Sphinx; LISA Laser). Fragments were washed out with irrigation. 5Fr DJ stent was placed antegrade at the end of the procedure.

RIRS: RIRS was performed using 4.9/7.9Fr URF-P7, flexible ureteroscope (Olympus, Germany), after placing 9.5/1 1.5F access sheath (Cook Medical). Stones were dusted using

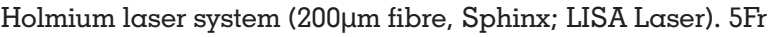
DJ stent was placed post procedure.

All patients were called for review after one month and were evaluated with X-ray KUB and Ultrasound scan to look for residual stones. Patient who had residual calculus underwent ureterorenoscopy for fragment clearance.

\section{Statistical Analysis:}

Results were statistically analyzed using the SPSS $®$ statistical software package, version 13.0. Data were presented as number (\%), mean \pm SD or mean and range. Between groups comparison was performed using student $t$ test for continuous variables and Chi square test for categorical variables. $P$ value of $<0.05$ was considered to be statistically significant.

\section{RESULTS}

Total of 168 patients with renal stones were admitted in our hospital over a period from January 2018 and April 2019, but only 66 patients met our inclusion criteria and were alternatively allotted to be treated with RIRS $(n=33)$ and $\operatorname{miniPCNL}(\mathrm{n}=33)$.

Patient's baseline demographics like age, gender distribution, stone size and location were all comparable in both the groups (Shown in Table 1).

Table 1. Patients Demographics.

\begin{tabular}{|l|c|c|}
\hline \multicolumn{1}{|c|}{ Variable } & RIRS & miniPCNL \\
\hline Number & 33 & 33 \\
\hline \multicolumn{1}{|c|}{ Memale } & $28(84.8)$ & $25(75.8)$ \\
& $05(15.2)$ & $08(24.2$ \\
\hline Age, years & $49.15 \pm 16.41$ & $44.97 \pm 13.14$ \\
& $(21.0-88.0)$ & $(23.0-70.0)$ \\
\hline Stone size, mm & $13.82 \pm 3.03$ & $12.52 \pm 2.99$ \\
& $(9.0-20.0)$ & $(8.0-20.0)$ \\
\hline Stone location & & $09(27.3)$ \\
Pelvis & $10(30.3)$ & $07(21.2)$ \\
Upper calyx & $09(27.3)$ & $07(21.2)$ \\
Mid calyx & $07(21.2)$ & $10(30.3)$ \\
Lower calyx & $07(21.2)$ & \\
\hline
\end{tabular}

Data presented as $n(\%)$ or mean $\pm S D$-Standard deviation (range).

The mean operative duration was longer in the RIRS group $58.3 \pm 14.51 \mathrm{~min}(38-90 \mathrm{~min})$ compared to the miniPCNL group $37.12 \pm 11.06 \mathrm{~min}(18-62 \mathrm{~min})$, which was statistically significant $(p<0.0001)$ (Shown in Table 2, Fig. 1).

Table 2: Intra- \& Postoperative characteristics

\begin{tabular}{|l|c|c|c|}
\hline \multicolumn{1}{|c|}{ Variables } & \multicolumn{1}{|c|}{$\begin{array}{c}\text { RIRS } \\
\mathbf{n}=33\end{array}$} & $\begin{array}{c}\text { miniPCNL } \\
\mathbf{n}=33\end{array}$ & $\begin{array}{c}\text { Statistical } \\
\text { Significance }\end{array}$ \\
\hline $\begin{array}{l}\text { Operative } \\
\text { duration (min) }\end{array}$ & $\begin{array}{l}58.3 \pm 14.51(38- \\
90)\end{array}$ & $\begin{array}{l}37.12 \pm 11.06 \\
(18-62)\end{array}$ & $<0.0001, \mathrm{~S}$ \\
\hline $\begin{array}{l}\text { Stone } \\
\text { learance, \% }\end{array}$ & 90.9 & 93.9 & NS \\
\hline $\begin{array}{l}\text { Blood } \\
\text { transfusion }\end{array}$ & 0 & $1(3.0)$ & NS \\
\hline Fever & $3(9.1)$ & $2(6.1)$ & NS \\
\hline $\begin{array}{l}\text { Postoperative } \\
\text { pain, VAS }\end{array}$ & $3.09 \pm 0.8(2-5)$ & $5.6 \pm 1.1(4-8)$ & $<0.0001, \mathrm{~S}$ \\
\hline $\begin{array}{l}\text { Postoperative } \\
\text { stay, days }\end{array}$ & $1.8 \pm 0.46(1-3)$ & $3.3 \pm 0.7(2-5)$ & $<0.0001, \mathrm{~S}$ \\
\hline
\end{tabular}

Data presented as mean $\pm S D$ (range) or $n(\%)$.

Visual Analogue Scale (0-10), S- significant, NS- not significant

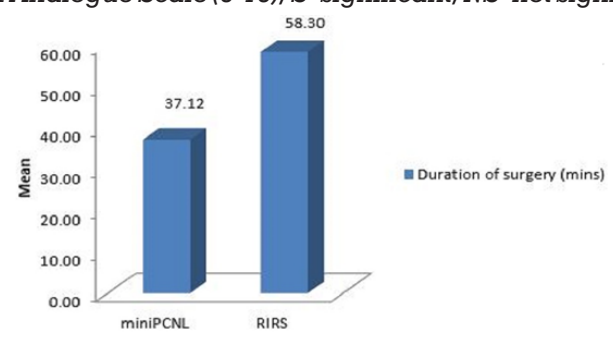

Fig l: Graphical representation of duration of surgery

Though stone clearance rate was marginally better in the miniPCNL group (93.9\%) compared to that achieved in the RIRS group (90.9\%), the difference between was statistically not significant $(\mathrm{p}=0.642)$ (Shown in Table 2, Fig. 2).

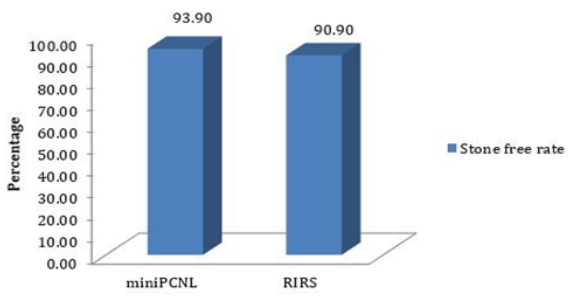

Fig 2: Graphical representation of stone free rate

The patients in the RIRS group experienced reasonably less pain (mean VAS score 3.09) postoperatively compared to those who underwent miniPCNL (mean VAS score 5.67), when measured on the visual analogue scale which was statistically significant $(p<0.0001)$ (Shown in Table 2, Fig. 3).

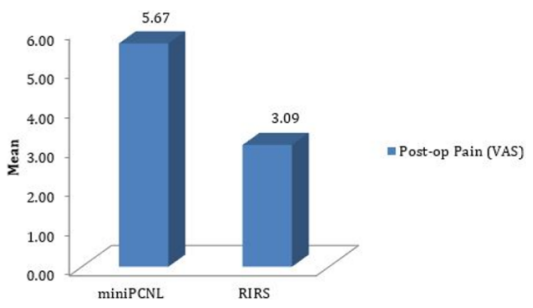

Fig 3: Graphical representation of post operative pain

In our study duration of post-operative stay for miniPCNL was longer with mean duration of 3.3days when compared to RIRS which was 1.8 days ( $p<0.0001$ ) (Shown in Table 2, Fig. 4). 


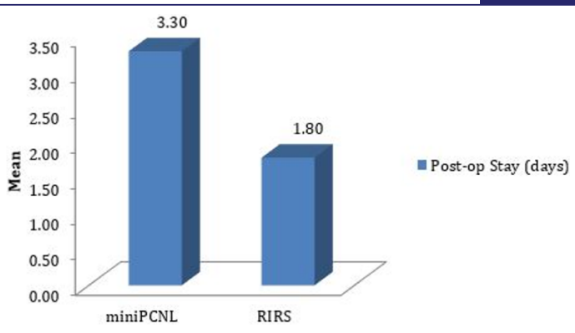

Fig 4: Graphical representation of post operative hospital stay

In terms of postoperative complications, one patient in the miniPCNL group $(3.0 \%)$, required blood transfusion. Two patients $(6.1 \%)$ in the miniPCNL group and three patients (9.1\%) in the RIRS group had postoperative fever; all were managed conservatively with antibiotics, which were titrated after two days as per urine culture and sensitivity report. These postoperative complications were found to be statistically insignificant.

Of the three patients in the RIRS group who had a residual fragment $(4-5 \mathrm{~mm})$, all had them in the proximal or mid ureter, of which two patients' fragment came out with the stent during stent removal and one patient required semirigid URS (ureterorenoscopy) and basketing for stone fragment removal. In the miniPCNL group, two patients had residual fragments at the end of one month $(4-6 \mathrm{~mm})$. Of these, one patient who had the stone fragment in the proximal ureter underwent semirigid URS with basketing for stone clearance and the other patient stone fragment came out with the stent.

\section{DISCUSSION}

RIRS in recent years has emerged as an alternative treatment option for renal calculi. Innovations in ureteroscopy has made possible unrestricted access to calculi at virtually all locations in the urinary tract. ${ }^{[10]}$ Failed ESWL and the inability to undergo ESWL (i.e., due to pregnancy, coagulopathy or morbid obesity) are recognized as indications for ureteroscopy ${ }^{[1]}$ For renal stones measuring less than $2 \mathrm{~cm}$, the stone clearance rate for RIRS was comparable to that for SWL as a primary procedure and good clearance rate has been achieved following the failure of SWL. ${ }^{[12]}$

Flexible ureteroscopes have limitations of narrow irrigation and working channels. When compared to older flexible ureteroscopes, newer instruments have excellent deflection. ${ }^{[13]}$ It has advantage of low morbidity like SWL along with added advantage of stone free rate (SFR) similar to PCNL in moderate and small sized renal calculi. Moreover, factors such as the BMI of the patient, stone density, lower pole anatomy and previous SWL might favour flexible ureteroscopy in certain cases. Flexible ureteroscopy is more useful in specific circumstances like calculi in horse shoe kidney or calyceal diverticulum, where SFR is lower in SWL. ${ }^{[1]}$

Since classical PCNL is typically employed for large stone burden, mini-PCNL and micro-PCNL have flourished as safe treatment options for smaller stones, especially when flexible instruments are not readily available. As such, practice patterns surrounding percutaneous surgery are evolving, and with that it is imperative to evaluate its benefits and limitations in comparison to RIRS.

In this study, we compared the outcomes of RIRS and mini$\mathrm{PCNL}$ for renal stones less than or equal to $2 \mathrm{~cm}$ size.

Stone free rate in our study was $90.9 \%$ in RIRS group as compared with $93.9 \%$ in miniPCNL group which was statistically not significant $(p=0.642)$. Our results were comparable with study by Fatih Akbulut et $\mathrm{al}^{[14]}$ and found to have similar result with $85.7 \%$ stone clearance rate in RIRS group and $90.3 \%$ in miniPCNL group which was not significant statistically ( $p$ 0.53). Similar results were observed from studies conducted by Sabnis et $\mathrm{al}^{[15]}$, Kirac M et al ${ }^{[16]}$ and Altaf Khan et $\mathrm{al}^{[17]}$ with no significant difference in stone free rate in both groups.

We noticed that operative time was higher in RIRS group $(58.3 \pm 14.51 \mathrm{~min})$ as compared to miniPCNL group (37.12 $\pm 11.06 \mathrm{~min}),(\mathrm{p}<0.0001)$. Studies by Sabnis et $\mathrm{al}^{[15]}$ and Altaf Khan et al ${ }^{[17]}$ also showed longer operative time for RIRS $(50.63 \pm 19.21$ and $70.2 \pm 10.3 \mathrm{~min}$ ) when compared to mini PCNL(40.81 $\pm 13.79 \mathrm{~min}$ and $38.7 \pm 9.8 \mathrm{~min})$ with significant $\mathrm{p}$ value of 0.003 and $<0.001$ respectively.

In our study post-operative hospital stay was less in RIRS group (1.8 \pm 0.46 days) when compared to miniPCNL group ( $3.3 \pm 0.7$ days) $(p<0.0001)$.Similar results were observed in studies conducted by Altaf Khan et $\alpha l^{[17]}$ and Pan J et al ${ }^{[18]}$ which showed significantly less hospital stay in RIRS (2.0 1.0 days and $1.9 \pm 1.3$ days) group compared to miniPCNL $(3.5 \pm 1.3$ days and $4.4 \pm 1.4$ days) group. Study done by Fatih Akbulut et $\mathrm{ll}^{[14]}$ also showed results with less hospital stay in RIRS group (22.4 \pm 18.2 hours) as compared with miniPCNL group $(63.8 \pm 32.1$ hours) which was statistically significant $(\mathrm{p}<0.001)$.However, study done by Sabnis et $\mathrm{al}^{[15]}$ showed no statistical difference in duration of post-operative stay between RIRS and miniPCNL group $(p=0.24)$

Post-operative pain (VAS score) was significantly more in miniPCNL group $(5.6 \pm 1.1)$ as compared to RIRS group $(3.09 \pm 0.8)$ which was statistically significant $(p<0.0001)$.Our results were comparable with study conducted by MeyyappanK et al ${ }^{[19]}$ which showed higher post-operative pain in miniPCNL group $(5.6 \pm 1.9)$ as compared to RIRS group $(3.76 \pm 1.39)$ which was statistically significant $(p<0.0001)$. Similar results were observed in studies conducted by Sabnis et al ${ }^{[15]}$ and Altaf Khan et al ${ }^{[17]}$ which showed significant higher pain score in miniPCNL(4.26 \pm 1.26 and $5.4 \pm 2.1)$ group compared with RIRS group(3.16 \pm 1.17 and $3.16 \pm 1.17$ ).

Post-operative fever (Clavien-Dindo Grade I) was observed in $9.1 \%$ of patients in RIRS group and $6.1 \%$ in miniPCNL group which was not statistically significant. Our results were comparable with study done by Zeng $\mathrm{G}$ et $\mathrm{al}^{[20]}$ and found to have similar result in both RIRS and miniPCNL group (13.5\% vs $8.8 \%$ ) which was statistically not significant. Similar results were observed from studies conducted by Resorlu B et $\mathrm{al}^{[21]}$ and Altaf Khan et al ${ }^{[17]}$ which showed no significant difference observed with incidence of post-operative fever in both groups.

In our study only one patient (3\%) in miniPCNL group received blood transfusion in post-operative period where as in RIRS group none of them needed blood transfusion, however this was statistically not significant. Study done by Meyyappan K et $\mathrm{al}^{[19]}$ showed $6 \%$ of patients received blood transfusion in miniPCNL group whereas none of the patients in RIRS group received blood transfusion which was not significant statistically. Similar results were found from studies by Resorlu B et $\mathrm{al}^{[2]]}$ and Altaf Khan et al ${ }^{[17]}$ with no significant difference between both groups with respect to post-operative blood transfusion.

LIMITATION: Study has not taken into consideration of costing for both procedures, which is the limitation in this study.

CONCLUSION: Though RIRS and miniPCNL have good and comparable stone free rate with low complication rates, favourable pain score and less hospital stay makes RIRS a preferred option over miniPCNL. 
REFERENCES:

1. Romero V, Akpinar H, Assimos DG. Kidney stones: A global picture of prevalence, incidence, and associated risk factors. Rev Urol 2010;12:e86-96

2. Clark JY, Thompson IM, Optenberg SA. Economic impact of urolithiasis in the United States. J Urol 1995; 154:2020-4

3. Türk C, Knoll T, Petrik A, Sarica K, Skolarikos A, Straub M, etal. Guidelines on Urolithiasis. Uroweb 2013. Available from:

4. http://www.uroweb.org/gls/pdf/21_Urolithiasis_LR.pdf.

5. Fernström I, Johansson B. Percutaneous pyelolithotomy. A new extraction technique. Scand J Urol Nephrol 1976; 10:257-9.

6. de la Rosette J. Assimos D, Desai M, Gutierrez J, Lingeman J, Scarpa R, et al. The Clinical Research Office of the endourological society percutaneous nephrolithotomy global study: Indications, complications, and outcomes in 5803 patients. J Endourol 2011;25:11-7.

7. Yamaguchi A, Skolarikos A, Buchholz NP, Chomón GB, Grasso M, Saba P, et al. Operating times and bleeding complications in percutaneous nephrolithotomy: A comparison of tract dilation methods in 5,537 patients in the Clinical Research Office of the endourological society percutaneous nephrolithotomy global study. J Endourol 2011;25:933-9.

8. Mishra S, Sharma R, Garg C, Kurien A, Sabnis R, Desai M. Prospective comparative study of miniperc and standard PNL for treatment of 1 to $2 \mathrm{~cm}$ size renal stone. BJU Int 201 1;108:896-9.

9. Michel MS, Trojan L, Rassweiler JJ. Complications in percutaneous nephrolithotomy. Eur Urol 2007;51:899-906.

10. Jackman SV, Docimo SG, Cadeddu JA, Bishoff JT, Kavoussi LR, Jarrett TW. The "mini-perc" technique: A less invasive alternative to percutaneous nephrolithotomy. World J Urol 1998:16:371-4.

11. AbuGhazaleh LA, Shunaigat AN, Budair Z. Retrograde intrarenallithotripsy for small renal stones in prepubertal children. Saudi J Kidney Dis Transpl 2011;22:492-6

12. Grasso M, Loisides P, Beaghler M, Bagley D. The case for primary endoscopic management of upper tract calculi: I. A critical review of 121 extracorporeal shock-wave lithotripsy failures. J Urology 1995;45:363-71.

13. Ho CC, Hafidzul J, Praveen S, Goh EH, Bong JJ, Lee BC, et al. Retrograde intrarenal surgery for renal stones smaller than $2 \mathrm{~cm}$. Singapore Med J 2010;51:512-5.

14. Fatih Akbulut, Onur Kucuktopcu, Emre Kandemir, Erkan Sonmezay, Abdulmuttalip Simsek, Faruk Ozgor, Murat Binbay, Ahmet Yaser Muslumanoglu, Gokhan Gurbuz. Comparison of flexible ureterorenoscopy and mini percutaneous nephrolithotomy in treatment of lower calyceal stones smaller than $2 \mathrm{~cm}$.Renal failure, 2016 doi.org/10.3109/0886022 x.2015.1128792

15. Sabnis, R. B., Jagtap, J., Mishra, S. and Desai, M. (2012), Treating renal calculi $1-2 \mathrm{~cm}$ in diameter with minipercutaneous or retrograde intrarenal surgery: $\alpha$ prospective comparative study. BJU International, 110: E346-E349. doi:10.1111/j.1464 410X.2012.11089.x

16. Kirac M, Bozkurt OF, Tunc L, Guneri C, Unsal A, Biri H. Comparison of retrograde intrarenal surgery and mini-percutaneous nephrolithotomy in management of lower-pole renal stones with a diameter of smaller than 15 mm. Urolithiasis. doi: 10.1007/s00240-013-0552-0.

17. Altaf Khan, Ashish Verma, Mujeebu Rahiman, Nischith Dsouza. Retrograde intrarenal surgery vs minipercutaneous nephrolithotripsy for lower pole stones less than $2 \mathrm{~cm}$. International Journal of Biomedical Research, [S.I.], v. 7, n. 11, p. 795-798, nov. 2016. ISSN: 0976- 9633.

18. Pan J, Chen $Q$, Xue W, Chen Y, Xia L, Chen H, et al. RIRS versus mPCNL for single renal stone of $2-3 \mathrm{~cm}$ : clinical outcome and cost-effective analysis in Chinese medical setting. Urolithiasis. 2013 Feb;4l(1):73-8. doi: 10.1007/s00240-012-0533-8

19. Meyyappan K, Philipraj SJ, Shet V Vishnuvardhan, Kone K (2018) Is RIRS better than Mini PCNL in Renal Calculus of size 1-2cms? A Prospective Randomised Comparative Study in a Tertiary Care Teaching Hospital. J Urol Ren Dis: JURD-189.

20. Zeng G, Zhu W, Li I, Zhao Z, Zeng T, Liu C, et al. The comparison of minimally invasive percutaneous nephrolithotomy and retrograde intrarenal surgery for stones larger than $2 \mathrm{~cm}$ in patients with a solitary kidney: a matched-pair analysis. World J Urol. 2014 Oct 21.

21. Resorlu B, Unsal A, Tepeler A, Atis G, Tokatli Z, Oztuna D, et al. Comparison of retrograde intrarenal surgery and mini-percutaneous nephrolithotomy in children with moderate-size kidney stones: results of multi-institutional analysis.JUrology.2012Sep;80(3):519-23. 